\title{
User-driven RFID applications and challenges
}

\author{
Aikaterini Mitrokotsa - Quan Z. Sheng • \\ Zakaria Maamar
}

Published online: 1 September 2011

(c) Springer-Verlag London Limited 2011

Radio Frequency IDentification (RFID) systems represent a key technology for ubiquitous computing that have attracted considerable attention in recent years. Their use has revolutionized diverse application areas including supply chain management, retail, anti-counterfeiting, luggage handling, and healthcare. Many organizations are planning to embrace or have already embraced RFID in their main operations to improve service quality, thwart product counterfeiting and theft, increase productivity, and maintain quality standards in many areas. Although RFID systems have many advantages, they also face significant challenges and vulnerabilities that need to be overcome in order to achieve a wider and more successful adoption of RFID technology.

This special issue presents the latest developments, trends, and research solutions for RFID technology. We have received 38 submissions. Ten papers were selected after several rounds of review by the guest editors and invited reviewers. These papers cover a wide range of topics in RFID research that reflect some key directions in this active yet diverse research area.

\footnotetext{
A. Mitrokotsa $(\square)$

Security and Cryptography Laboraroty (LASEC),

School of Computer and Communication Sciences,

EPFL, Lausanne, Switzerland

e-mail: katerina.mitrokotsa@epfl.ch

Q. Z. Sheng

School of Computer Science, The University of Adelaide, Adelaide, Australia

e-mail: qsheng@cs.adelaide.edu.au

Z. Maamar

College of Information Technology, Zayed University,

Dubai, UAE

e-mail: zakaria.maamar@zu.ac.ae
}

The paper by Asadzadeh et al. proposes a gesture recognition technique based on RFID tags. More precisely, they propose an approach which uses RFID communication in order to recognize hand gestures with high accuracy.

Some papers proposed techniques for making applications of RFID communication more robust in environments with high levels of noise or interference. More precisely, the paper by Kim et al. proposes a location sensing system based on active RFID technology in order to enhance the worker's safety under a cargo crane in the steel industry. In order to guarantee the efficiency of the location sensing system under the high levels of interference in the environment, the authors develop circular polarization antennas and a three-dimensional location sensing algorithm.

The paper by $\mathrm{Wu}$ et al. proposes a localization algorithm based on genetic algorithms in order to estimate the location of unknown nodes (RFID tags).

The paper by Darcy et al. proposes some algorithms that can be used to clean and enhance the quality of the data generated by the communication between RFID readers and RFID tags.

The paper by Schapranow et al. proposes an architecture for storing and searching pharmaceutical RFID event data. Additionally, they provide qualitative requirements for software components developed for RFID and provide a quantitative analysis of operational costs for a dedicated service provider for anti-counterfeiting.

The paper by Sanchez Lopez et al. investigates the technologies that will be fundamental for realizing the Internet of Things (IOT) concept and proposes an architecture that integrates all of them into a single platform. The paper by Angerer et al. introduces the concept of rapid prototyping in RFID and provides a survey of system simulators, demonstrators, and rapid prototyping environments. Additionally, they propose a set of guidelines for 
the setup of such a rapid prototyping system applicable to RFID technology.

Security and privacy are serious concerns in RFID applications. Batina et al. investigate the use of public key encryption on RFID authentication protocols and propose a privacy-preserving multi-party grouping proof protocol which relies exclusively on the use of elliptic curve cryptography. Additionally, a novel ECC hardware architecture designed for RFID is presented to illustrate the implementation feasibility of the proposed solutions.

The paper by Erguler et al. investigates the problem of RFID tag delegation while the paper by Peris-Lopez et al. proposes the use of a secure RFID authentication scheme based on cryptographic puzzles in order to avoid the disclosure of confidential information without requiring an online database.

We thank all authors for considering this special issue for publishing their research results in the area of RFID. We would like to thank the referees who provided very useful and thoughtful feedback to the authors. This special issue would not have been possible without their help and selfless dedication. We also would like to thank the Editor in Chief of the Journal of Personal and Ubiquitous Computing, Prof. Peter Thomas, for his continuous support during the review and publication process.

Finally, we hope that this collection of papers can help shed some light and generate fruitful discussions on current and future RFID research and developments. 